\title{
Designing a dictionary of patterns of destructive utterances in the task of identifying destructive information influence
}

\author{
E.P. Okhapkina, ${ }^{1,2,}$, V.P. Okhapkin ${ }^{3}$, A.O. Iskhakova ${ }^{4}$, and A.Y. Iskhakov ${ }^{4}$ \\ ${ }^{1}$ Bauman Moscow State Technical University, 2nd Baumanskaya St., 5, Moscow, 105005, Russia \\ ${ }^{2}$ Russian State University for the Humanities, Miusskaya square, 6, Moscow, 125993, GSP-3, Russia \\ ${ }^{3}$ Center of Expert and Analytical and Information Technologies of Accounts Chamber of the Russian \\ Federation, Zubovskiy blvd., 21-23b2, Moscow, 119021, Russia \\ ${ }^{4}$ V.A. Trapeznikov Institute of control sciences of Russian Academy of Sciences, Profsoyuznaya st., \\ 65, building 1, Moscow, 117342, Russia
}

\begin{abstract}
Due to the high level of tension in modern society, social networks are widely used for destructive management of the information space. This aspect of the use of social networks has become particularly important in the light of events taking place in the world (Hong Kong, Syria, France and Ukraine). According to statistics, about $50 \%$ of politicized active groups of social networks are subjects to targeted control actions aimed at spreading negative moods in the political sphere. The escalation of conflicts in society generates the most dangerous type of destructive information influence (DII) that require rapid, large-scale coordination of participants in order to attract new supporters and their organizations. Massive DII on the participants of social networks groups exacerbated the problem of promptly identifying the facts of influence, and created serious prerequisites for the development and improvement of methods and means of identifying DII in social networks. The relevance of this problem is due to the existence of a number of methodological and technological problems in the subject area under consideration, one of them is the lack of patterns of network messages containing elements of DII. In the study, the authors consider an approach to designing a dictionary of patterns of destructive utterances.
\end{abstract}

\section{Introduction}

In the context of a large amount of information presented in social networks, an effective approach to solving the problem of identifying DII is to create a system for analyzing network messages. It is worth noting that network messages can include various types of content (text, audio, photos, geotags, etc.). In this case, the content can be presented as one of the types or a combination of types, but in this study, the authors will only consider messages containing text. Like any text analysis system, a DII detection system should include a set of dictionaries, such as a morphological dictionary, a synonym dictionary, tonal labeled dictionaries, and a

\footnotetext{
* Corresponding author: okhapkina.ep@bmstu.ru
} 
number of others. Since the object of message analysis is a verbal expression containing signs of DII, for an adequate conclusion about the utterance, it is necessary to include a dictionary of patterns of destructive utterances in the set of dictionaries. Since such dictionaries are not available in the public domain and are not presented in scientific research, the authors propose an approach to building such a dictionary based on the categorical apparatus for constructing ontologies.

\section{An approach to designing a dictionary of destructive utterances}

The content of network messages can be viewed from the standpoint of a cognitive approach. This approach is associated with understanding how a person "decodes" information about reality, including information obtained by reading text messages, and how he organizes it, for example, for making comparisons, decisions and etc. This allows you to identify the fact that the authors of the messages are pursuing certain goals. From this point of view, messages from social networks, including those containing information management, can be considered as anthropotexts, i.e. as a textual realization of a personality of a particular linguistic personality type, for example, political, social, psychological, gender, cultural, etc. Thus, the actors of influence, represented by one of the types of linguistic personality, from the point of view of the cognitive approach, implement certain scenarios for exerting informational influence. Scenarios are a typical data structure that reflects a certain type of conflict (social, ethnic, political, etc.) and are expressed in certain speech forms - schemes of communicative influence. In accordance with the characteristics of mass communication, most of the texts of the media and social networks in particular are addressed to a wide range of readers, who, like the actors of influence, are linguistic personalities. Realizing a certain type of scenario, the actor of influence must take into account the interests and needs of different linguistic personalities-addressees. Texts of messages or articles presented in social networks written in this way can be interpreted by recipients from different positions. Thus, messages of a typical structure containing DIV attributes can be represented as a domain knowledge model.

The starting point for creating any domain knowledge model is to choose its categorical apparatus. For the correct use of the same information from dictionaries and thesauri also used in other text analysis systems, it is necessary to adopt a unified conceptualization. The conceptualization is based on the categories of abstractions that are associated with the construction of the term underlying any ontology. The analysis of various ontologies [1-5], existing at the present time, showed that basically the categories of abstractions of ontological models are different. In this regard, the work proposes a categorical apparatus on the basis of the works of linguists, logicians and informatics [3,5-10], as well as the approach proposed in the work [3].

The dictionary of patterns of destructive utterances, like many other dictionaries, is based on the concept of a term as a sign. Since the pattern of a destructive utterance should reflect one or another type of informational influence, then in addition to the term-concept describing object / subject of speech, the pattern should also contain rules for the connection of the term-concept with actions, states, events (an integral part of the description of any managerial influence), and may also contain descriptions of properties and quantities. We define patterns as a collection of lexical rules expressing destructive informational impact. The advantage of using patterns is their universalism in relation to various genres and topics of the analyzed texts. In this case, only the dictionary of negative labeled words requires updating. It is obvious that the development of patterns is a laborious process, but their number is countable and finite. The more patterns, the greater the coverage of extracted phrases and phrases when analyzing text messages. 
The figure 1 represents conceptual knowledge of the term. To build a unified thesaurus, it is important not only to determine the lexical composition of the terminology, but also the set of used relations. If the semantic structure is defined as a network of semantic relations connecting units of knowledge, then the semantic relations introduced into the thesaurus should model this structure.

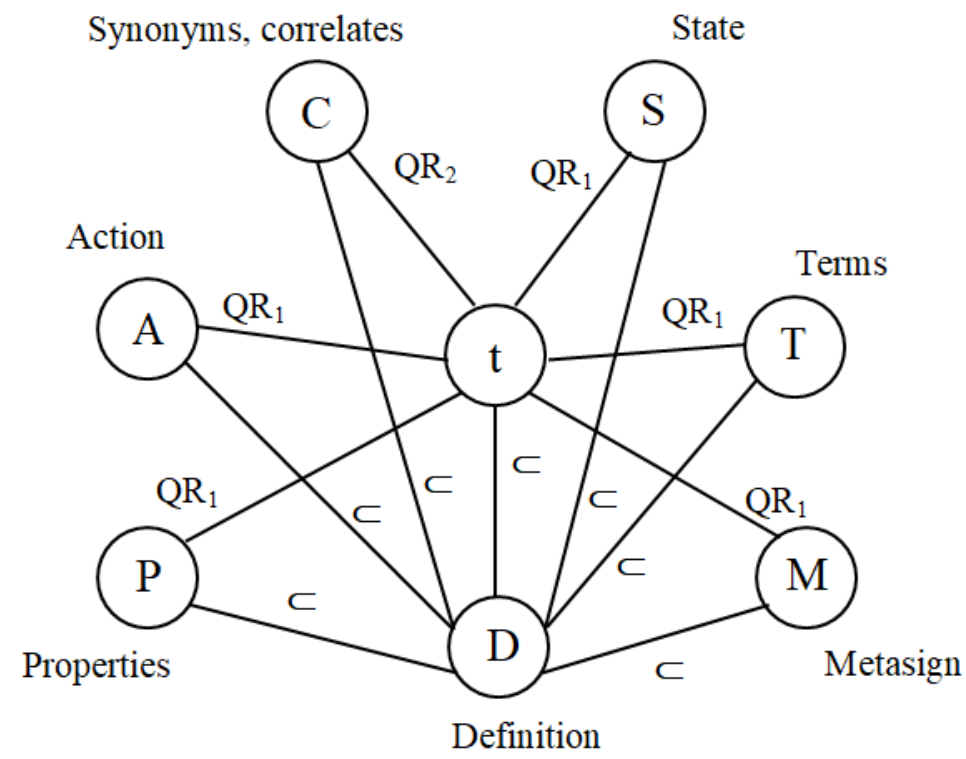

Fig. 1. Graphic interpretation of the "Term" as a sign: $\mathrm{t}$ - term; QR1 - quality relationships; QR2 quantitative relations, İ - inclusion relation.

The role of the sign is played by the term $t$, shown on the graph at the vertex of the same name. The vertex $\mathrm{T}$ defines the set of terms $\mathrm{T}$ that are related to the term $\mathrm{t}$. In this case, the meaning of the term is reflected by the vertices $\mathrm{D}, \mathrm{C}, \mathrm{P}, \mathrm{S}$, and $\mathrm{T}$, the actions associated with the sign are reflected by the vertex $\mathrm{A}$, and the metasign is reflected by the vertex $\mathrm{M}$. The arcs of the graph correspond to the classification of I. Dalberg [6], detailed on the basis of the works of linguists S. E. Nikitina [7], N. N. Lavrova [7] and others [3]. The vertex D is introduced in the construction, since the term can have several interpretations. To reflect the pragmatic aspect of the term, the sign construction introduces the vertex A, which is necessary to represent knowledge about actions related to the object. Thus, the proposed approach to constructing the sign structures of Dahlberg's conceptual objects, as the main categories of abstractions, makes it possible to create a unified conceptualization of the subject area. In accordance with the sign construction shown in figure 1, the dictionary entry "Term" can be represented as a tuple:

Concept $=<\mathrm{t}, \mathrm{D}, \mathrm{P}, \mathrm{A}, \mathrm{C}, \mathrm{S}, \mathrm{T}, \mathrm{M}>$,

$\mathrm{t}$ :

$\mathrm{t}=<\mathrm{t} 1, \mathrm{t} 2, \mathrm{t} 3>$ :

$\mathrm{t} 1$ - the name of the term;

t2 - the type of the conceptual object "Term";

t3 - type of entity: material, non-material.

D:

$\mathrm{D}=\{\mathrm{di} \mid \mathrm{di}-$ the substantial definition, $\mathrm{i}=1 \div \mathrm{n}, \mathrm{n}-$ number of selected definitions $\}$.

$\mathrm{P}$ : 
$\mathrm{P}=\{(\mathrm{p} 1, \mathrm{p} 2) \mathrm{i} \mid \mathrm{p} 1$ - property name; $\mathrm{p} 2$ - link to a dictionary entry describing $\mathrm{p} 1 ; \mathrm{i}-$ number of concept properties $\}$.

A:

$A=\{(a 1, a 2) i \mid$ a 1 - action; a 2 - link to a dictionary entry describing a1 as a term; $\mathrm{i}=1 \div \mathrm{n}$, $\mathrm{n}-$ number of actions relevant to the term $\}$.

C:

A set of terms that have a quantitative relationship with the term $t$, described by a deuce $<\mathrm{C} 1, \mathrm{C} 2>$, where $\mathrm{C} 1=\{(\mathrm{c} 11, \mathrm{c} 12) \mathrm{i} \mid \mathrm{c} 11$ - synonym; $\mathrm{c} 12-$ link to a dictionary entry describing $\mathrm{c} 11$ as a term; $\mathrm{i}=1 \div \mathrm{k}, \mathrm{k}-$ number of synonyms $\}, \mathrm{C} 2=\{(\mathrm{c} 21, \mathrm{c} 22) \mathrm{i} \mid \mathrm{c} 21$ - correlate; $\mathrm{c} 22-$ link to a dictionary entry describing $\mathrm{c} 21$ as a term; $\mathrm{i}=1 \div \mathrm{m}, \mathrm{m}-$ number of correlates $\}$.

S:

$\mathrm{S}=\{(\mathrm{s} 1, \mathrm{~s} 2) \mathrm{i} \mid \mathrm{s} 1-$ name of the term describing the state of the entity, s2 - link to a dictionary entry describing the term $\mathrm{s} 1, \mathrm{i}=1 \div \mathrm{n}, \mathrm{n}=|\mathrm{S}|\}$.

T:

The set of concepts (terms) that have a qualitative relationship with the term $\mathrm{t}$.

M:

This element is used to define relationships between sign systems. It is described by a deuce $<$ M1, M2 $>$, where M1 - a method of metalanguage representation that allows to fix the relationship between the term and its representation in the metalanguage; M2 - a term in a different language that allows you to record the relationship between terms in different subject areas. Each element M1 and M2 also consists of two components, the first of which is the name of the term (a method of metalanguage representation or a term in another language), and the second is a link to the dictionary article that describes this term.

The dictionary entry "Term" is central. When describing a term using the elements $\mathrm{P}, \mathrm{A}$, and $\mathrm{S}$, a link is established with dictionary entries describing properties, actions, and states. The elements $\mathrm{C}$ and $\mathrm{T}$ establish various relationships with other terms. The $\mathrm{M}$ element allows you to establish relationships with terms in a related subject area.

Next, we present the construction of the "Action" sign (figure 2) based on the explicit action model [3], which combines the casual and propositional models:

Action $=<\mathrm{a}, \mathrm{D}, \mathrm{P}, \mathrm{SO}, \mathrm{C}, \mathrm{I}, \mathrm{A}, \mathrm{E}, \mathrm{M}>$,

- action name;

$\mathrm{SO}$ - subject or object of the action;

I - the instrument used to perform the action;

$\mathrm{A}-\mathrm{a}$ set of actions that are in a qualitative relationship with the action a;

$\mathrm{E}$ - the event when the action occurs, D, P, C, have a value similar to the corresponding "Term", since actions, like terms, can form hierarchies. They may consist of separate procedures or procedures from operations. They can also have types or variants.

In accordance with the sign construction shown in figure 2, the dictionary entry "Action" is described as follows:

a:

$\mathrm{a}=<\mathrm{a} 1, \mathrm{a} 2, \mathrm{a} 3>, \mathrm{a} 1$ - the name of the term, a2 - type of the concept object "Action", a3 type of action, selected from the list: process, procedure, function, operation, way, method.

$\mathrm{D}$ :

$\mathrm{D}=\{\mathrm{Di} \mid \mathrm{Di}-$ the substantial definition, $\mathrm{i}=1 \div \mathrm{n}, \mathrm{n}-$ number of selected definitions $\}$.

P:

$\mathrm{P}=\{(\mathrm{p} 1, \mathrm{p} 2) \mathrm{i} \mid \mathrm{p} 1$ - property name; $\mathrm{p} 2$ - link to a dictionary entry describing $\mathrm{p} 1 ; \mathrm{i}-$ number of action properties $\}$.

SO:

$\mathrm{SO}=<\mathrm{SO} 1, \mathrm{SO} 2, \mathrm{SO} 3>$, SO1 - type: object or subject; $\mathrm{SO} 2$ - name of the term that is the object or subject of the action; SO3 - link to a dictionary entry describing this term $\mathrm{SO} 2$.

I: 
$\mathrm{I}=<\mathrm{I} 1, \mathrm{I} 2>, \mathrm{I} 1-$ name of the term that identifies the instrument used to perform the action, the article describing the term I1.

E:

$\mathrm{E}=<\mathrm{E} 1, \mathrm{E} 2>$, where $\mathrm{E} 1$ - name of the action being performed; describing the term $\mathrm{E} 1$.

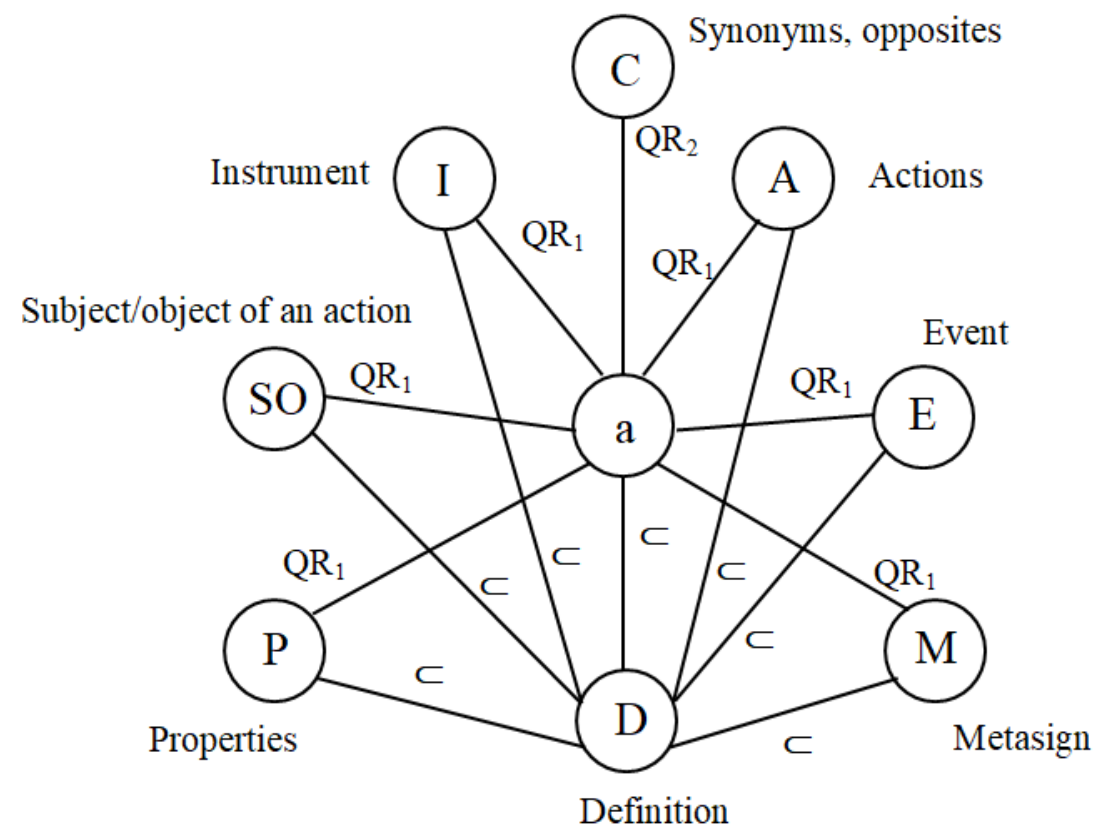

Fig. 2. Graphic interpretation of the "Action" as a sign: a - action, QR1 - qualitative relations, QR2 quantitative relations, and İ - inclusion relation.

All data about an action, such as action parameters, initial object or initial data of an action, final object or results of an action, derived object or intermediate data of an action, location of action, etc., are described in the element $\mathrm{P}$ - action properties.

It is worth noting that the description of the properties (details) of the performed action allows a better performance of the procedure for detecting DII in network messages. For example, the following types of actants in the control model can be distinguished in a message:

To be the "agent" of the action;

To be the "object" of the action;

To be the "addressee" of the action;

To be the "theme" of the action;

To be an "instrument" of action;

To be the "place" of action;

To be the "starting point" of the action;

To be the "end point" of the action;

To be the "target" of the action;

To be a "condition" for an action;

To be the "time" of action;

To be the "way" of action;

To be the "expiration date";

To be the "amount" of the action;

To be the "result" of an action; 
To be the "counterparty" of the action;

In addition to the "Action" category, sign categories such as "State" and "Event" are responsible for representing the dynamic part of the domain model. In the works of linguists, they are referred to functional-semantic categories of dynamism [1-10]. Sign constructions for them are also based on the works of S. E. Nikitina and Dalberg and are tuples.

Such a multi-level description of the constituent patterns of destructive information influence allows you to create a set of scenarios for organizing destructive management distributed in social networks and provide a different combination of the categories of signs described above when implementing a scenario based on logical rules and relationships between signs. Each scenario, in turn, is an element of the subject area and is described as an ontology class.

$$
O=\langle X, R, F\rangle,
$$

where $X$ - a finite set of domain concepts provided by an ontology $O ; R-$ a finite set of relationships between domain concepts; $F$-a finite set of interpretation functions defined on ontology concepts and/or relationships $O$ The interpretation function can be a verbal description of the term (abstract), a formula for calculating the meaning of the term, an algorithmic description, or a definition in the form of a logical formula.

To develop a dictionary of destructive utterance patterns, the Protégé 5.5.0 ontoreditor was used. The set of domain concepts $\mathrm{X}$ includes words that make up destructive domain patterns. Words include nouns, verbs, adjectives, auxiliary verbs, definitions combined with nouns, and other parts of speech. As an example, figure 3 shows an ontology fragment that includes three patterns of destructive utterances (Mod_1, Mod_2, Mod_3). Each of the patterns includes a set of parts of speech and a set of lexical rules $\mathrm{R}$ for combining them to form a pattern, as well as a set of interpretation functions $\mathrm{F}$.

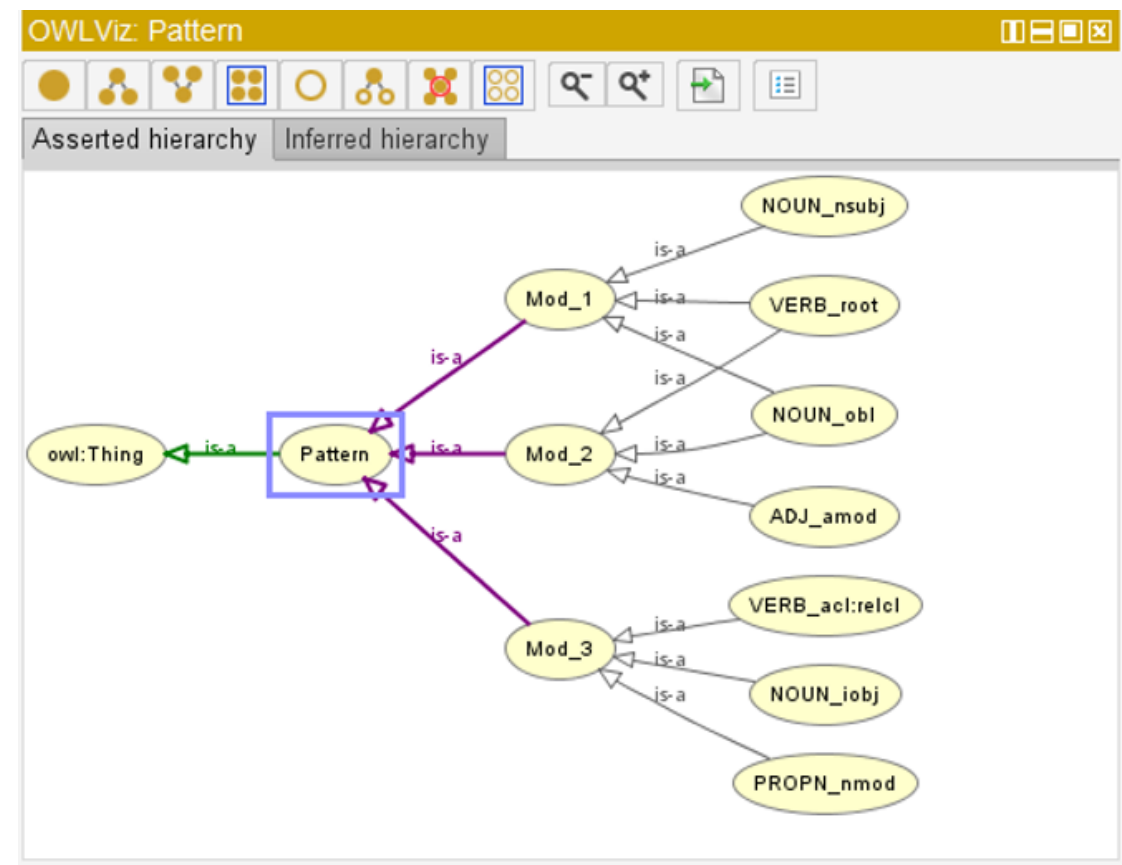

Fig. 3. Fragment of the ontology of patterns of destructive utterances.

Figure 4 shows an ontograph representing one of the particular variants of a combination of words that activate the potential presence of a destructive pattern in the message text. It is 
also worth noting that as a part of the lexical rule for each word, the label or range of possible tonal labels of the word is indicated (see Fig. 4, the red floating inscription).

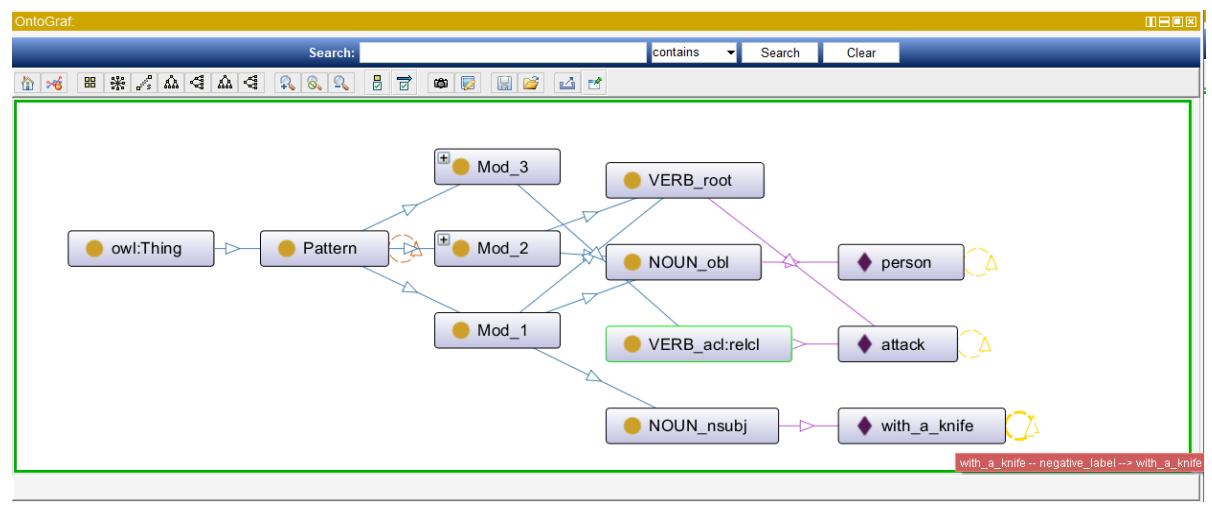

Fig. 4. An example of a particular graph structure of a pattern.

\section{Conclusion}

Thus, the described ontology can be used to identify information influence. It is obvious that in the context of identifying aggressive, destructive information, the key role is played by the dictionary of marked-up negative words. It is the combination of "dictionary-pattern" that increases the ability to accurately identify a negative utterance and its semantic boundaries. It is important to note that the construction of patterns requires the fulfillment of the uniqueness condition - the pattern should be focused on extracting phrases and utterances only of a certain morphological construction.

\section{Acknowledgments}

The reported study is funded by RFBR according to the research project No. 18-29-22104 "Development of socio-cyberphysical system of monitoring of diverse Internet-content for counteraction to manifestation of aggression, pressure and other forms of destructive impact on individual and group consciousness of users".

\section{References}

1. Dobrov B and Lukashevich N 2006 Computational linguistics and intellectual technologies: Proc of Int conf (Dialogue) p 138

2. Asai K 1993 Applied Fuzzy Systems (Moscow: Mir)

3. Naykhanova L 2008 Software products and systems $\mathbf{2}$

4. Smirnov A 2002 Artificial Intelligence News 13

5. Biryukov B 1965 The logical structure of scientific knowledge (Moscow: Nauka) p 91

6. Dahlberg I 2006 Ko Knowledge Organization 33(1) 11

7. Nikitina S and Slyusareva N 1987 Semantic analysis of the language of science: on the material of linguistics (Moscow: Nauka)

8. Iskhakova A Meshcheryakov R 2017 RPC 2017 - Proc of the 2nd Russian-Pacific Conference on Computer Technology and Applications (Institute of Electrical and Electronics Engineers Inc., Vladivostok) p 85 
9. Iskhakova A 2019 Proc of the VIth Int Workshop 'Critical Infrastructures: Contingency Management, Intelligent, Agent-Based, Cloud Computing and Cyber Security' (IWCI 2019) (Atlantis Press, Irkutsk) p 14

10. Shumskaya A 2016 11th International conference interactive systems "Interactive systems: problems of human - computer interaction", Collection of scientific papers (U1yanovsk: Ulyanovsk state technical University) p 211

11. Abramyan M Litovchenko D 2019 Procs of Eighth China-Russia Conf (Southern Federal University, I.I. Vorovich Institute of Mathematics, Mechanics, and Computer Science) p 97

12. Vo A 2019 Semantic and syntactic analysis in learning representation based on a sentiment analysis model, Applied intelligence 